\title{
Loss of placental growth factor ameliorates maternal hypertension and preeclampsia in mice
}

\begin{abstract}
Jacqueline G. Parchem, ${ }^{1,2,3}$ Keizo Kanasaki, ${ }^{4}$ Megumi Kanasaki, ${ }^{4}$ Hikaru Sugimoto, ${ }^{1,4}$ Liang Xie, ${ }^{4}$ Yuki Hamano, ${ }^{4}$ Soo Bong Lee, ${ }^{4}$ Vincent H. Gattone, ${ }^{5}$ Samuel Parry, ${ }^{6}$ Jerome F. Strauss, ${ }^{7}$ Vesna D. Garovic, ${ }^{8}$ Thomas F. McElrath, ${ }^{9}$ Karen H. Lu, ${ }^{10}$ Baha M. Sibai, ${ }^{2}$ Valerie S. LeBleu, ${ }^{1,4}$ Peter Carmeliet, ${ }^{11,12}$ and Raghu Kalluri ${ }^{1,4}$

'Department of Cancer Biology, Metastasis Research Center, University of Texas MD Anderson Cancer Center, Houston, Texas, USA. ²Department of Obstetrics, Gynecology and Reproductive Sciences, McGovern Medical School, The University of Texas Health Science Center at Houston, Houston, Texas, USA. ${ }^{3}$ Department of Obstetrics and Gynecology, Baylor College of Medicine, Houston, Texas, USA. ${ }^{4}$ Division of Matrix Biology, Department of Medicine, Beth Israel Deaconess Medical Center and Harvard Medical School, Boston, Massachusetts, USA. ${ }^{5}$ Department of Anatomy and Cell Biology, Indiana University School of Medicine, Indianapolis, Indiana, USA. ${ }^{\circ}$ Department of Obstetrics and Gynecology, Perelman School of Medicine, University of Pennsylvania, Philadelphia, Pennsylvania, USA. Department of Obstetrics and Gynecology, Virginia Commonwealth University School of Medicine, Richmond, Virginia, USA. ${ }^{8}$ Department of Internal Medicine, Mayo Clinic, Rochester, Minnesota, USA. ${ }^{9}$ Department of Obstetrics and Gynecology, Brigham and Women's Hospital, Boston, Massachusetts, USA. ${ }^{10}$ Department of Gynecologic Oncology, University of Texas MD Anderson Cancer Center, Houston, Texas, USA. "Laboratory of Angiogenesis and Vascular Metabolism, Department of Oncology, Katholieke Universiteit (KU) Leuven, Leuven, Belgium. ${ }^{22}$ Laboratory of Angiogenesis and Vascular Metabolism, Vesalius Research Center, Center for Cancer Biology (CCB), Vlaams Instituut voor Biotechnologie (VIB), Leuven, Belgium.
\end{abstract}

\begin{abstract}
Preeclampsia remains a clinical challenge due to its poorly understood pathogenesis. A prevailing notion is that increased placental production of soluble fms-like tyrosine kinase-1 (sFlt-1) causes the maternal syndrome by inhibiting proangiogenic placental growth factor (PIGF) and VEGF. However, the significance of PIGF suppression in preeclampsia is uncertain. To test whether preeclampsia results from the imbalance of angiogenic factors reflected by an abnormal sFlt-1/PIGF ratio, we studied PICF KO $\left(\mathrm{Pgf}^{--}\right)$mice and noted that the mice did not develop signs or sequelae of preeclampsia despite a marked elevation in circulating SFLT-1. Notably, PICF KO mice had morphologically distinct placentas, showing an accumulation of junctional zone glycogen. We next considered the role of placental PIGF in an established model of preeclampsia (pregnant catechol-0-methyltransferase-deficient [COMT-deficient] mice) by generating mice with deletions in both the Pgf and Comt genes. Deletion of placental PIGF in the context of COMT loss resulted in a reduction in maternal blood pressure and increased placental glycogen, indicating that loss of PICF might be protective against the development of preeclampsia. These results identify a role for PICF in placental development and support a complex model for the pathogenesis of preeclampsia beyond an angiogenic factor imbalance.
\end{abstract}

\section{Introduction}

Preeclampsia is a common and serious hypertensive disorder affecting approximately $5 \%$ of pregnancies. The underlying biology of the disease is complex and not fully understood (1). Poor invasion of the placenta, characterized by incomplete remodeling of uterine spiral arteries early in pregnancy, is thought to promote the altered production of placenta-derived factors in the latter half of pregnancy in response to hypoxia that results from the vascular remodeling defect (2-4). These factors induce maternal endothelial activation and inflammation, which manifest clinically as hypertension and varying degrees of end-organ injury. Significant attention has been given to the role of the circulating angiogenic factors soluble fms-like tyrosine kinase-1 (sFlt-1, also known as soluble VEGFR-1) and placental growth factor (PlGF) in this process as well as their potential utility as early biomarkers of the disease (5-8). In pre-

Authorship note: VHG is deceased.

Conflict of interest: The authors have declared that no conflict of interest exists. License: Copyright 2018, American Society for Clinical Investigation.

Submitted: December 4, 2017; Accepted: August 28, 2018.

Reference information: / Clin Invest. 2018;128(11):5008-5017.

https://doi.org/10.1172/JCI99026. eclampsia, elevated sFlt-1 - a truncated, soluble form of Flt-1 lacking the tyrosine kinase domain - acts as a decoy receptor, functioning as a molecular sponge to limit signaling induced by elevated levels of circulating proangiogenic ligands and preventing their action on target cells (9-11). In rodents, adenovirus-mediated overexpression of sFlt-1 or treatment with anti-VEGF antibodies or recombinant sFlt-1 induces hypertension, proteinuria, and kidney glomerular injury in pregnancy (11-13). These observations provided the foundation for a widely accepted model for preeclampsia in which an angiogenic factor imbalance, characterized by an abnormally high ratio of sFlt-1 to PlGF and VEGF, is thought to be an essential component of disease pathogenesis (reviewed in refs. 1, 2, 14).

PlGF, a member of the VEGF family, was originally discovered in the placenta, where it was proposed to regulate vascular development and trophoblast growth and differentiation (15). Unlike VEGF, which is required for angiogenesis and endothelial cell maintenance and binds both Flt-1/sFlt-1 and Flk-1, PlGF is redundant for vascular development and selectively binds Flt-1/ sFlt-1 (16-18). PlGF stimulates endothelial cell growth, migration, and survival and plays a central role in pathologic angiogenesis, including in cancer and tissue ischemia $(19,20)$. In addition to signaling through Flt-1, PlGF promotes angiogenesis by competing 
A

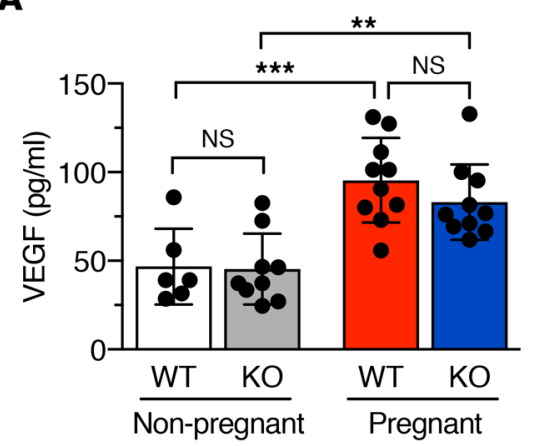

C

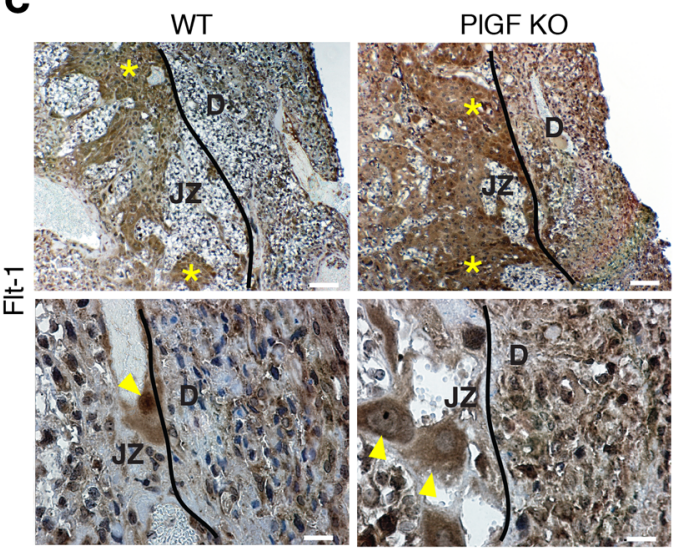

B

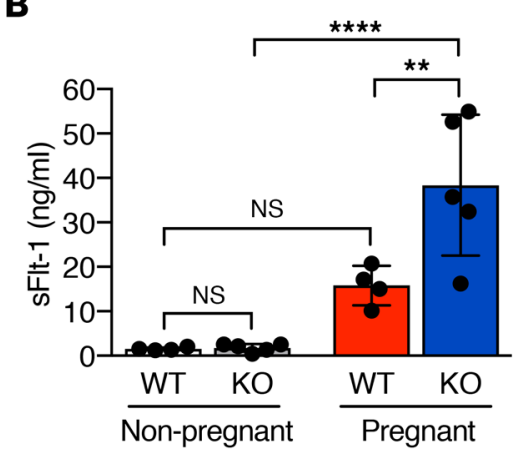

D

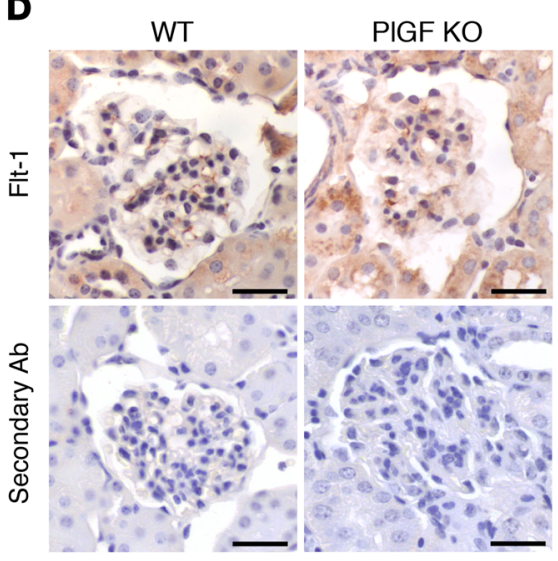

Figure 1. Elevation of serum sFlt-1 and placental Flt-1 levels in pregnant PIGF KO mice. (A) Serum VEGF levels measured by ELISA in nonpregnant WT $(n=6)$ and PICF KO $(n=9)$ and pregnant WT $(n=10)$ and PIGF KO $(n=10)$ mice. (B) Serum sFlt-1 levels (ELISA) in nonpregnant and pregnant WT $(n=4)$ and PICF $\mathrm{KO}(n=5)$ mice. (C) Immunohistochemistry for Flt-1 shows increased Flt-1 protein expression in PIGF KO placentas and decidua compared with WT. Spongiotrophoblast cells marked by yellow asterisks, parietal trophoblast giant cells by yellow arrowheads. Solid lines mark the border between the junctional zone and the decidua. $D$, decidua; JZ, junctional zone. Scale bars: $100 \mu \mathrm{m}$ (top row); $25 \mu \mathrm{m}$ (bottom row). (D) Immunohistochemistry for Flt-1 in the kidney glomerulus showing similar expression in PIGF KO and WT (upper panels). Secondary antibody-only control shown in lower panels. Scale bars: $25 \mu \mathrm{m}$. Results are shown as mean \pm SD. One-way ANOVA. ${ }^{* *} P<0.01,{ }^{* *} P<$ $0.001,{ }^{* * *} P<0.0001$. with VEGFA for Flt-1, freeing VEGFA to signal through Flk-1 (17). $\mathrm{PlGF}$ is upregulated in pregnancy, with levels peaking in the serum of pregnant women in the early third trimester $(10,21,22)$. In pregnant mice, PlGF has been shown to influence maternal cardiovascular physiology (23). In the placenta, it is expressed in trophoblast giant cells and decidual natural killer cells $(21,24)$. Although it is dispensable for embryogenesis (16), recent data support its function in fetal cerebrovascular development (25). Despite interest in the utility of PlGF for clinical diagnostics, the role of PlGF in preeclampsia and in placental development is not well understood.

To delineate the pathogenic role of sFlt- 1 and PlGF in preeclampsia, we used PlGF KO mice to create an angiogenic factor imbalance in pregnancy. We also examined the role of PlGF in preeclampsia by generating mice with genetic deletion of both PlGF and the enzyme catechol-O-methyltransferase (COMT). Reduced COMT activity and low plasma levels of its metabolic product, 2-methoxyestradiol (2-ME), are observed in preeclampsia (26-32). Mechanistically, 2-ME has been shown to promote extravillous trophoblast invasion (33) and migration (29) and to dysregulate HIF$1 \alpha$ function (34-36). Treatment of first trimester placental villous explants with 2-ME rescued altered levels of sFlt-1 and hypoxiainducible factor 1- $\alpha$ (HIF-1 $\alpha$ ) induced by hypoxia (36). We previously reported that pregnant COMT KO mice develop a preeclampsia-like phenotype characterized by hypertension, proteinuria, renal glomerular endotheliosis, increased placental hypoxia, an influx of decidual natural killer cells, and elevated circulating sFlt-1 (37). Treatment of pregnant COMT KO mice with 2-ME rescued the preeclampsia-like phenotype, while treatment of WT mice with an inhibitor of COMT (Ro41-0960) mimicked the COMT KO phenotype (37). Additional findings consistent with preeclampsia have been reported in various studies of pregnant COMT-deficient mice, including fetal growth restriction $(37,38)$, abnormal uterine artery Doppler waveforms (38), an exaggerated response to angiotensin II (39), and an abnormal metabolome (40). We hypothesized that PlGF deficiency would induce a preeclampsia-like phenotype by promoting an antiangiogenic state defined by excess sFlt- 1 and that loss of PlGF in the setting of COMT deficiency would exacerbate the phenotype. Our findings, however, suggest that PlGF is protective against the development of preeclampsia-like features in COMT KO mice and that placental PlGF plays a role in normal placental development and metabolism.

\section{Results}

Loss of PlGF in pregnancy results in elevated sFlt-1 levels. We used mice with genetic deletion of PlGF ( $P g f /$; PlGF KO) to evaluate the effect of PlGF deficiency on serum angiogenic factor levels in pregnancy. PlGF expression examined by immunofluorescence was confirmed in placentas of WT mice, but not PlGF KO mice, with expression primarily in parietal trophoblast giant cell and spongiotrophoblast populations, consistent with prior studies (data not shown; refs. 21, 24). Serum VEGF and sFlt-1 levels were measured in PlGF KO and WT mice by ELISA and were found to be elevated in pregnant compared with nonpregnant mice, as expected (Figure 1, A and B). Whereas serum VEGF levels in pregnant KO and WT mice were similar (Figure 1A), sFlt-1 levels were significantly elevated in pregnant PlGF KO mice compared with pregnant WT (Figure 1B). Immunostaining for Flt-1 revealed increased Flt-1 expression in the junctional zone spongiotrophoblasts, parietal trophoblast giant cells, and asso- 
A

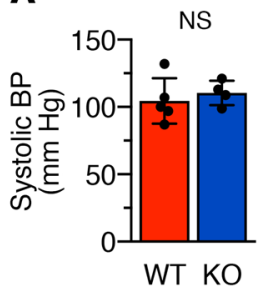

E
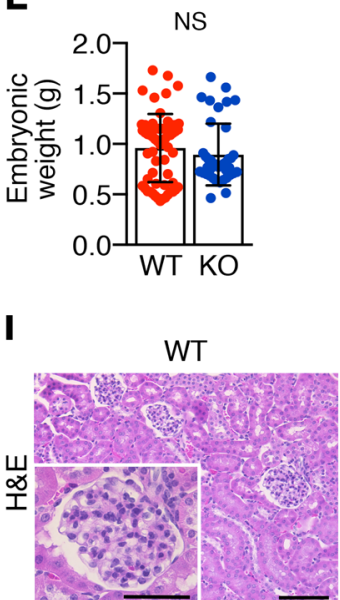

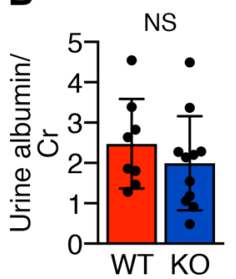

$\mathbf{F}$

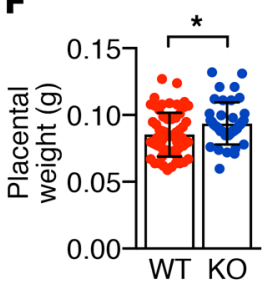

C

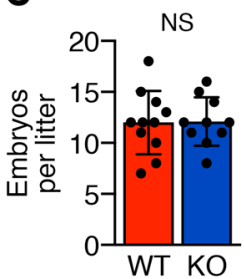

G

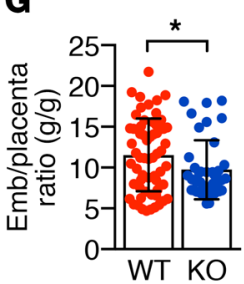

D

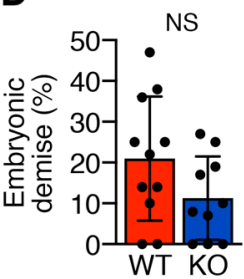

H

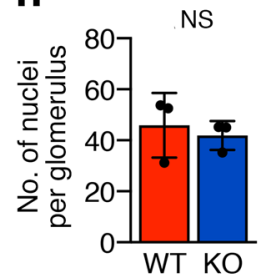

J

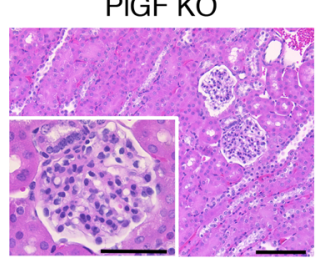

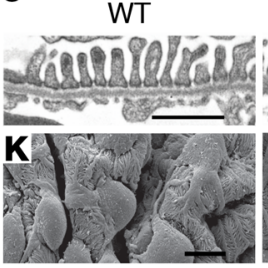

PIGF KO

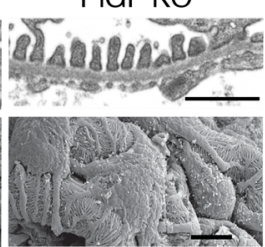

Figure 2. Pregnant PIGF KO mice do not develop signs or complications of preeclampsia. (A) Systolic blood pressure (BP) of pregnant WT ( $n=5)$ and PIGF KO $(n=4)$ mice. (B) Proteinuria measured by spot urine albumin/creatinine $(\mathrm{Cr})$ ratios for pregnant WT $(n=8)$ and KO mice $(n=11)$. Blood pressure and urine protein measured between E17 and E20. (C) Total number of embryos per litter, includes resorbing embryos ( $n=10$ litters). (D) Percentage of resorbed embryos per litter ( $n=10$ litters). (E-F) Weights of individual embryos (E) and placentas (F) from WT ( $n=63$ embryos/placentas from 7 litters) and KO ( $n=40$ embryos/ placentas from 4 litters) mice collected between E17 and E20. (C) Embryo/placenta ratio decreased in PIGF KO ( $n=40$ embryos from 4 litters) compared with WT ( $n=62$ embryos from 7 litters). (H) Number of nuclei per glomerulus ( $n=3$ mice, $6-10$ glomeruli per mouse). (I) Representative images of H\&E-stained kidney sections from the indicated groups showing normal tubules and glomeruli. Scale bars: $100 \mu \mathrm{m} ; 50 \mu \mathrm{m}$ (inset). (J) Normal podocyte foot processes, glomerular basement membrane, and slit diaphragms by transmission electron microscopy and (K) normal podocyte foot processes by scanning electron microscopy for both groups. Scale bars: $1 \mu \mathrm{m}(\mathrm{J}) ; 5 \mu \mathrm{m}$ (K). Results are shown as mean $\pm \mathrm{SD}$. Two-tailed, unpaired $t$ test. ${ }^{*} P<0.05$.

ciated decidua of KO placentas (Figure 1C and Supplemental Figure $1, \mathrm{~A}$ and B; supplemental material available online with this article; https://doi.org/10.1172/JCI99026DS1), but not in the kidneys of PlGF KO mice (Figure 1D). These results suggest that the elevated circulating sFlt-1 levels in pregnant PlGF KO mice originated from increased placental Flt-1 expression.

Pregnant PlGF KO mice do not develop signs of preeclampsia. Hypertension and proteinuria, the classic features of preeclampsia, were evaluated in pregnant PIGF KO and WT mice. Tail-cuff blood pressures measured at E17-E2O were similar for PlGF KO and WT mice (Figure 2A), as were urine albumin/creatinine ratios (Figure 2B). Preeclampsia is also associated with increased rates of fetal growth restriction and fetal demise due to placental insufficiency. Therefore, we compared the litter sizes, resorption rates, and embryonic weights and found no significant differences between KO and WT mice (Figure 2, C-E). These findings are in concordance with the previously reported normal fertility and litter size of PlGF KO mice $(16,23)$. The embryo/placenta ratio was significantly decreased in PlGF KO compared with WT (Figure 2G) due to increased placental weight in PlGF KO mice (Figure 2F and Supplemental Table 1).

Glomerular capillary endotheliosis and podocyte injury are signature kidney lesions associated with preeclampsia. Histological analyses of the maternal kidneys revealed normal kidney architecture in pregnant PlGF KO mice (Figure 2I). Detailed examination of glomerular structure also showed a similar number of cells per glomerulus (Figure $2 \mathrm{H}$ ) and no differences in renal corpuscle, glomerular tuft, or Bowman's space areas (Supplemental Figure 2, A and B). Transmission electron microscopy (Figure 2J) and scanning electron microscopy (Figure 2K) analyses revealed intact podocyte foot processes and absence of glomerular endotheliosis and hyperproliferation, indicating normal glomerular ultrastructure in pregnant PlGF KO mice. Collectively, these results show that PlGF KO mice do not show manifestations of preeclampsia despite an abnormal elevation in circulating sFlt-1.

PlGF KO placentas exhibit increased junctional zone glycogen and decreased labyrinth area. The mouse placenta is divided into 3 compartments: the decidua on the maternal side, the middle junctional zone, and the vessel-dense labyrinth layer on the fetal side. Alterations in the overall placental architecture were evident on histological analyses of H\&E-stained cross-sections, which revealed a significantly thicker junctional zone and thinner labyrinth layer in PlGF KO placentas (Figure 3, A and D). Expansion of the junctional zone appeared to be due primarily to increased numbers of vacuolated glycogen trophoblast cells (Figure 3A). Glycogen accumulation in PlGF KO placentas was confirmed 
WT
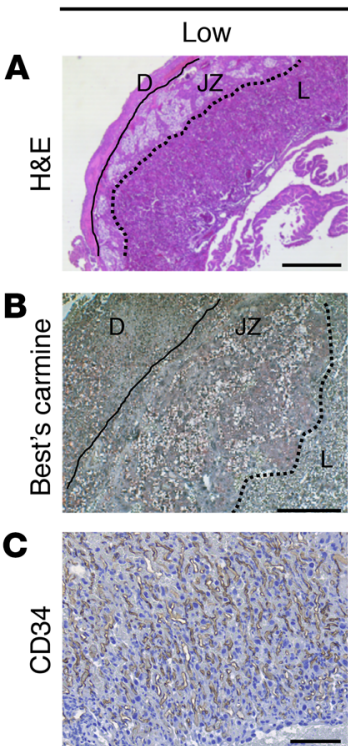
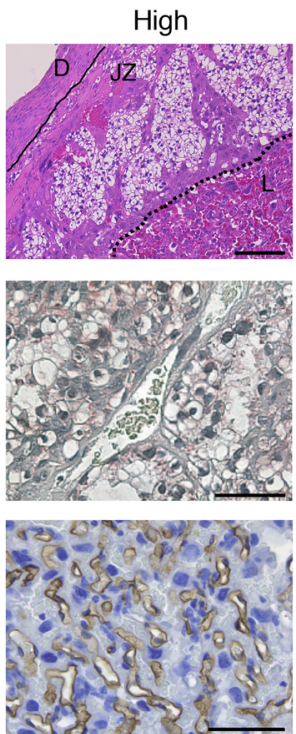

PIGF KO
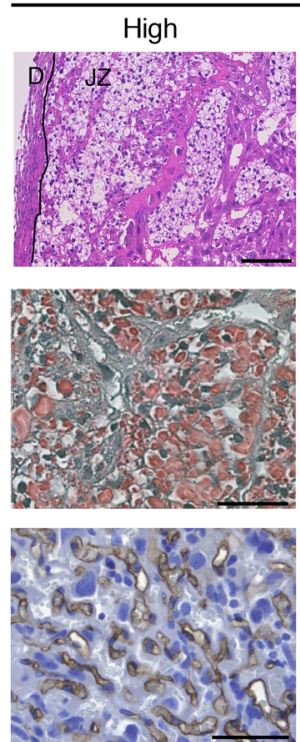
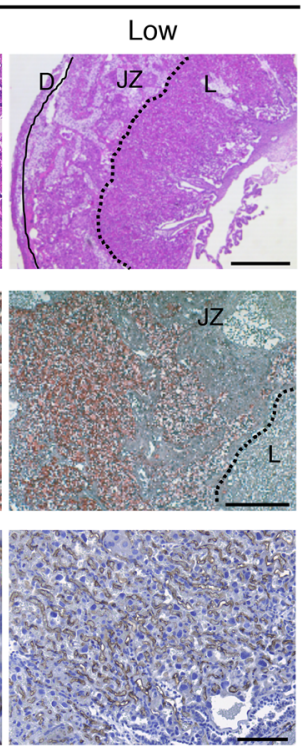

D

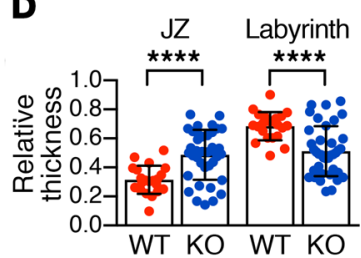

$\mathbf{E}$
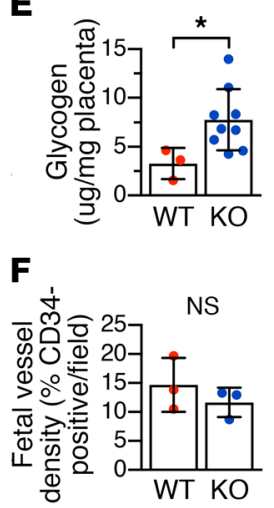

Figure 3. Changes in morphology and glycogen content in PIGF KO placentas. (A) Representative low- and high-magnification images of H\&E-stained cross-sections of placentas showing increased numbers of junctional zone glycogen trophoblast cells, which appear as islets of cells with clear cytoplasm due to the high content of glycogen-containing vacuoles. Scale bars: $500 \mu \mathrm{m}$ (low); $100 \mu \mathrm{m}$ (high). (B) Specific staining of glycogen with Best's carmine (red) in the junctional zone (E19). Scale bars: $200 \mu \mathrm{m}$ (low); $50 \mu \mathrm{m}$ (high). Solid and dotted lines mark the borders between the junctional zone and the decidua and junctional zone and labyrinth, respectively. (C) Immunohistochemistry for CD34 showing similar staining of fetal vasculature in the labyrinth. Scale bars: $100 \mu \mathrm{m}$ (low); $50 \mu \mathrm{m}$ (high). (D) Quantification of placental layer measurements expressed as a proportion of total placental thickness. At least 2 midplacental cross sections were analyzed from each placenta from WT ( $n=21$ placentas from 7 litters) and KO ( $n=38$ placentas from 5 litters) mice. (E) Quantification of relative glycogen content ( $\mu$ g glycogen/mg placental tissue) in WT $(n=3)$ and KO $(n=9)$ placentas. (F) Quantification of CD34 staining (\% positive area) of the labyrinth in WT and KO placentas ( $n=3$ placentas from 3 litters, 3 representative high-power fields per placenta) L, labyrinth. Results are shown as mean \pm SD $(\mathbf{D}, \mathbf{E})$ and mean \pm SEM $(\mathbf{F})$. Two-tailed, unpaired $t$ test. ${ }^{*} P<0.05$; ${ }^{* * * *} P<0.0001$.

with the glycogen-specific stain Best's carmine (Figure 3B) and quantification of tissue glycogen content (Figure 3E). Parietal trophoblast giant cells (demarcating the junctional zone-decidual border) and spongiotrophoblasts in the junctional zone appeared normal in PlGF KO placentas (Supplemental Figure 3, A and B).

We next examined the labyrinth layer, which comprises a dense network of fetal and maternal blood vessels. Comparisons of PlGF KO with WT placentas revealed a similar density of endothelial cell-lined fetal vessels identified by CD34 immunohistochemistry (Figure 3, C and F). Mononuclear sinusoidal trophoblast giant cells lining the maternal vessels had normal morphology by H\&E (Supplemental Figure 3C) and pan-cytokeratin staining (Supplemental Figure 3D). Overall, labyrinth vessel architecture was similar in PlGF KO and WT placentas, but labyrinth area in PlGF KO placentas was decreased compared with that in WT (Figure 3D). These placental changes were associated with increased placental weight in PlGF KO mice (Figure 2F). Collectively, these results show that PIGF loss leads to junctional zone glycogen accumulation, a reduction in labyrinth area, and a larger placenta, but does not significantly affect trophoblast morphology or labyrinth architecture.

Loss of PlGF ameliorates the preeclampsia-like phenotype in COMT-deficient mice. Since we observed an increase in sFlt-1 levels and a placental phenotype in PlGF KO mice, but an absence of preeclampsia manifestations, we hypothesized that loss of PlGF might be protective. We tested the effects of PlGF loss in preeclampsia using the COMT KO model, which develops a preeclampsia-like phenotype (37). PIGF KO and COMT KO mice were bred to generate double $\mathrm{KO}(\mathrm{DKO})$ mice $\left(\mathrm{Pgf} / ; \mathrm{Comt}^{-/}\right)$. DKO females were mated to COMT KO males (DKO ${ }^{\text {XPGF+ }}$ ) or DKO males (DKO ${ }^{\text {XDKO }}$ ) to assess the role of placental PlGF on the maternal preeclampsia phenotype. This mating scheme produced pregnancies with placentas expressing $\left(\mathrm{Pgf}^{+/} ; \mathrm{Comt}^{-/}\right.$, placental $\left.\mathrm{PlGF}^{+/-}\right)$or lacking (Pgf $/$; $\mathrm{Comt}^{-/}$, placental $\mathrm{PlGF}^{-/}$) PlGF, respectively (Figure 4A). Lack of placental PIGF in pregnant DKO ${ }^{\times D K O}$ mice was associated with a lower systolic blood pressure (Figure 4B) and a trend toward decreased proteinuria (Figure $4 \mathrm{C}$ ) when compared with DKO ${ }^{\mathrm{XPIGF}+}$ pregnant females, indicating partial rescue of the preeclampsia phenotype caused by COMT deficiency. The relative reduction in blood pressure in pregnant DKO mice lacking placental PlGF was associated with a nonsignificant elevation of sFlt-1 compared with that in pregnant $\mathrm{DKO}^{\mathrm{XPGF+}}$ mice (Figure $4 \mathrm{D}$ ), in which sFlt-1 levels approximate the levels observed in the COMT KO model of preeclampsia. Blood pressure, proteinuria, and sFlt-1 data for PlGF, COMT, and DKO mice are summarized in Supplemental Table 2. Similarly to PlGF KO litters, which show a decreased embryo/ placenta ratio, DKO ${ }^{\mathrm{DKKO}}$ litters also showed a decreased embryo/ placenta ratio (Figure $4 \mathrm{G}$ and Supplemental Table 1), which appeared to be driven by a significant reduction in embryonic weight (Figure 4E), as placental weight was not dependent on the PlGF genotype (Figure 4F).

Based on the findings in PlGF KO placentas, we anticipated that PlGF loss would lead to an increase in glycogen content 
A

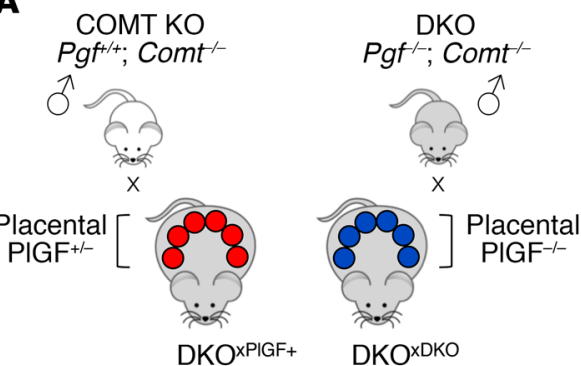

E

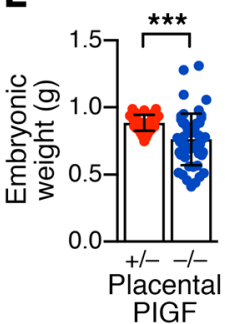

F

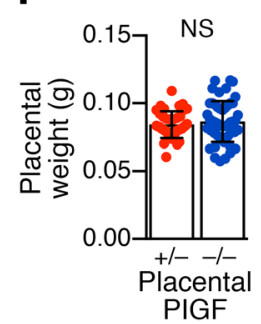

B

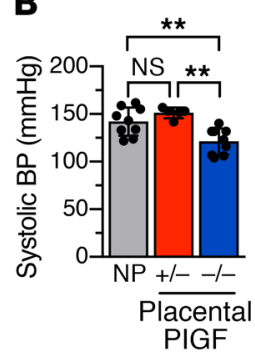

C

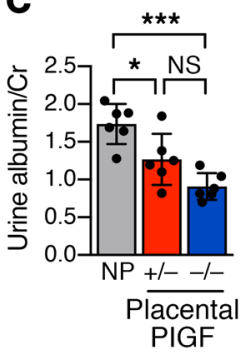

D

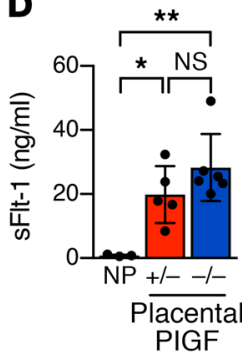

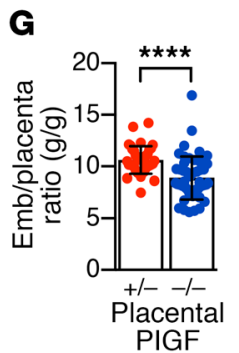

H

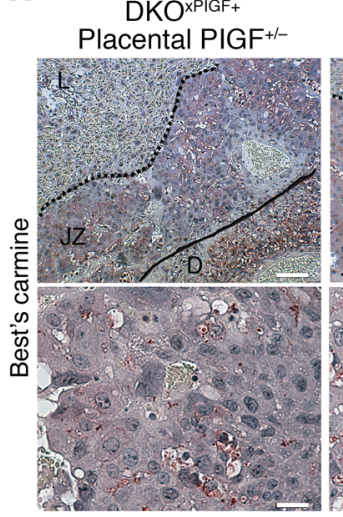

DKO XDKO Placental PIGF-/-
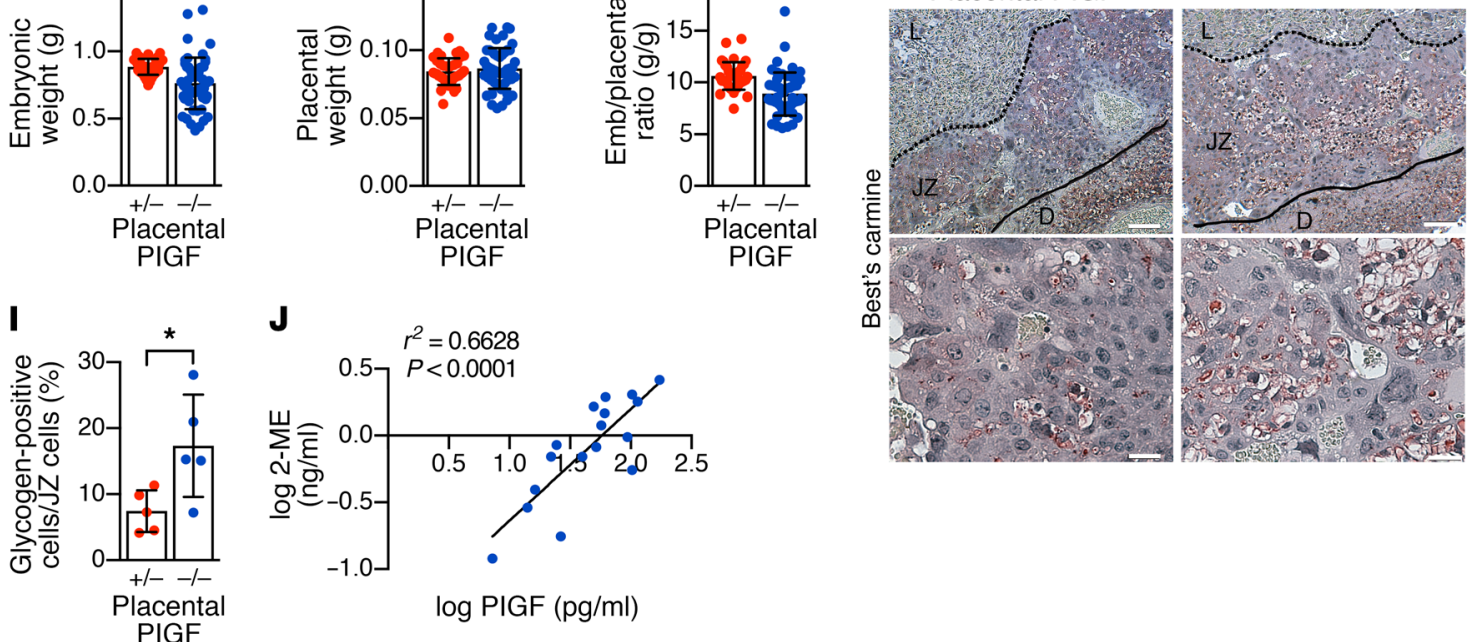

J

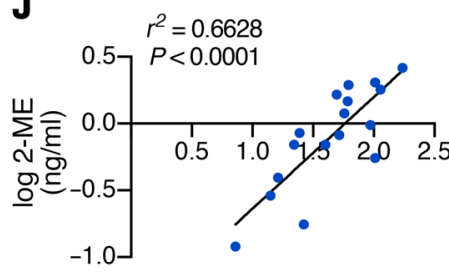

$\log$ PIGF $(\mathrm{pg} / \mathrm{ml})$

Figure 4. Loss of PIGF ameliorates preeclampsia-like phenotype in COMT KO mice. (A) Schematic of the breeding strategy and embryo/placenta genotypes. (B) Systolic blood pressure in nonpregnant DKO $\left(\right.$ Pgf $^{-1-} ;$ Comt $\left.^{-1-} ; n=9\right)$ and pregnant DKO XPIGF+ $(n=5)$ and DKO ${ }^{\times D K O}(n=9)$ mice at E17. (C) Proteinuria measured by spot urine albumin/creatinine ratio $(n=6)$. (D) Plasma sFlt-1 measured by ELISA in nonpregnant DKO $(n=3)$, DKO $\times$ PICF+ $(n=5)$, and DKOXKKO $(n=6)$ at E17. (E-F) Weights of embryos $(\mathbf{E})$ and placentas $(\mathbf{F})$ collected at E17 from DKOXPlGF+ $\left(n=38\right.$ embryos from 5 litters) and DKO ${ }^{\times D K O}(n=52$ embryos from 7 litters) cohorts. (G) Embryo/placenta ratio decreased in DKO ${ }^{\text {хKо }}$ (placental PIGF ${ }^{-I}$ ). (H) Representative images of the placenta and of the junctional zone showing Best's carmine glycogen staining of E17 placentas. Solid and dotted lines mark the borders between the junctional zone and the decidua and the junctional zone and labyrinth, respectively. Scale bars: $100 \mu \mathrm{m}$ (top row); $25 \mu \mathrm{m}$ (bottom row). (I) Ratio (\%) of glycogen-positive cells to total junctional zone cells per visual field ( $n=5$ placentas). (J) Linear regression analysis of the relationship between plasma PIGF and 2-ME levels in preeclampsia (human cases; $n=17$ ). PIGF samples here are also represented in Supplemental Figure 5C. NP, nonpregnant. Results are shown as mean \pm SD. One-way ANOVA (B-D), 2-tailed, unpaired $t$ test $(\mathbf{E}-\mathbf{H})$, or Pearson's correlation test $(\mathrm{J}) .{ }^{*} P<0.05 ;{ }^{* *} P<0.01 ;{ }^{* *} P<0.001 ;{ }^{* * *} P<0.0001$.

in DKO ${ }^{\mathrm{XDKO}}$ placentas. At baseline, COMT KO mice exhibited decreased placental glycogen that was rescued with 2-ME treatment (Supplemental Figure 4, A and B). In the DKO context, this loss of glycogen in COMT-deficient placentas was represented in the $\mathrm{DKO}^{\mathrm{XPIGF}}$ mice, which showed a comparable percentage of glycogen-positive cells in the junctional zone compared with COMT KO mice (Figure 4H and Supplemental Figure 4B). The glycogen content in DKO ${ }^{\times D K O}$ placentas lacking PlGF was indeed increased compared with that in $\mathrm{DKO}^{\mathrm{XPGF}}$ placentas, confirmed by Best's carmine stain, indicating partial rescue of the reduced glycogen phenotype seen in COMT KO placentas (Figure 4, $\mathrm{H}$ and I). In preeclamptic women, alterations in placental glycogen content have previously been reported (41-43). This was confirmed in our study by measuring tissue glycogen content in human placentas from normotensive control versus preeclamptic pregnancies (Supplemental Figure 5A).

Thickening of the basement membrane has also been associated with preeclampsia and other conditions of placental insufficiency, such as hypertension and fetal growth restriction $(44,45)$.
Thus, we examined placental expression of basement membrane proteins laminin and entactin (also known as nidogen-1) in DKO and COMT KO placentas. Both proteins were more abundant in PlGF-expressing placentas from $\mathrm{DKO}^{\mathrm{xPlGF}+}$ compared with $\mathrm{DKO}^{\mathrm{xDKO}}$ mice by immunofluorescence (Supplemental Figure 6A). Consistent with this finding, increased staining of these basement membrane proteins was observed in COMT KO placentas compared with WT or COMT KO treated with 2-ME (Supplemental Figure 6B). In summary, we have shown that PlGF loss in the COMT KO preeclampsia model leads to increased glycogen content and reduced basement membrane protein deposition in the placenta, which were associated with a reduction in maternal blood pressure and alterations in embryonic growth.

Correlation between PlGF and 2-ME in plasma from preeclamptic women. In the COMT KO mouse, low levels of 2-ME (metabolite of COMT enzyme activity) are associated with placental hypoxia and increased HIF-1 $\alpha$ expression (37). Because low PlGF levels are also strongly associated with placental hypoxia and preeclampsia, we evaluated the relationship between PlGF and 2-ME 
levels in human plasma. First, we validated previous findings by our group and others showing reduced 2-ME $(26-29,31,32)$ and PlGF levels $(6,10,11)$ in preeclamptic compared with control women (Supplemental Figure 5, B and C). Then we assessed their relationship in patient plasma and found a significant positive, linear correlation between PlGF and 2-ME among women with preeclampsia (Figure 4J).

\section{Discussion}

Numerous studies have established the association between an elevated sFlt-1/PlGF ratio and preeclampsia (5, 10, 11, 46-49). However, whether this biochemical imbalance is directly related to disease etiology remains largely unknown. We studied mice with genetic deletion of PlGF to address whether the resultant angiogenic factor imbalance would be sufficient to induce preeclampsia. We found that pregnant PlGF KO mice displayed an abnormally elevated level of circulating sFlt-1, but lacked manifestations of preeclampsia, in contrast with mice lacking COMT, despite a mean serum concentration of sFlt-1 that was greater than peak concentrations observed in human preeclampsia and in other rodent models of preeclampsia $(10,11)$. Upregulation of placental Flt-1 and sFlt-1 receptors was presumed to occur in response to ligand depletion. Notably, loss of placental PlGF in the COMT KO preeclampsia model ameliorated the maternal hypertension phenotype of those mice. Furthermore, deletion of PlGF altered placental morphology, supporting a role for PlGF in placental development.

Our study demonstrated that PlGF KO placentas have a distinct morphology characterized by enlargement of the junctional zone due to glycogen accumulation and a reduction in the area of the labyrinth compartment. Glycogen trophoblasts, a population of specialized trophoblast cells found in the junctional zone and decidua, are rich in glycogen-containing vacuoles and have been compared with invasive extravillous cytotrophoblasts of the human placenta $(50,51)$. Although the primary purpose of placental glycogen is a matter of debate, it is thought to serve as an important energy source for fetal growth and can be utilized to maintain placental function under hypoxic conditions (51-55). Many studies have reported alterations in placental metabolism and glycogen content in preeclampsia and fetal growth abnormalities in human and rodent pregnancy, although it is challenging to make definitive conclusions from these studies due to sample and methodological differences $(41-43,51,56)$. What is evident is that the significance of increased placental glycogen alterations is context dependent, for instance, indicating excess nutrient availability in diabetes as opposed to impaired utilization or compensation for placental dysfunction in preeclampsia. There is evidence that increased glycogen may serve a protective function. In cancer cells, for example, increases in cellular glycogen storage have been shown to maintain cellular ATP levels as a protective mechanism against bioenergetic stress (57). In genetic mouse models, placental glycogen has been shown to play a role in preserving fetal growth (53). Furthermore, increased placental glycogen in human preeclampsia cases was reported in conjunction with a significant increase in placental glycogen synthase activity, suggesting a metabolic state favoring glycogen synthesis in preeclampsia (41). Collectively, the literature suggests that placental glycogen effectively acts as a barometer for disturbances in fetoplacental metabolism and that its availability may be an important etiologic component of some pregnancy conditions and a consequence of others.

We found that loss of PlGF in both WT and COMT KO backgrounds resulted in a significant increase in placental glycogen and a reduction in the embryo/placenta ratio, a parameter used as a rough proxy for placental function $(58,59)$. We speculate that this phenotype represented a compensatory response to disturbances in placental function that have been documented in the COMT-deficient background (37), and are inferred in the PlGFsingle KO model based on increased sFlt-1 levels and the reduction in labyrinth area, which directly affects placental nutrient exchange capacity. The placental phenotype resulting from PlGF loss had a net beneficial effect for the mother, demonstrated by the absence of preeclampsia in pregnant PlGF KO mice and a reduction in blood pressure in pregnant $\mathrm{DKO}^{\mathrm{xDKO}}$. The change in labyrinth area was not entirely surprising, given the established role of PlGF in angiogenesis. Based on our histologic findings and the localization of Flt-1 receptor to trophoblasts residing in the junctional zone and at the decidual border (i.e., glycogen trophoblast cells, spongiotrophoblast cells, and endovascular trophoblast cells), we postulate that the location of PlGF action in the placenta is primarily the junctional zone (60). Whether PlGF directly regulates the activity of key enzymes involved in glycogen metabolism or whether additional mechanisms of compensation, such as local upregulation of VEGF, occurred is not known; these are areas of interest for future studies. Our findings suggest that PlGF loss had a protective effect potentially mediated through alterations in placental metabolism, which strongly correlates with placental glycogen content and preeclampsia $(41,42,51,56,61,62)$.

In plasma samples from preeclamptic women, we found a significant positive correlation between levels of 2-ME and PlGF. Reduced COMT activity and 2-ME have consistently been associated with preeclampsia and placental hypoxia and metabolism $(29,37,40,62-64)$. We speculate that suppression of PlGF in the context of low 2-ME levels in preeclampsia might promote placental glycogen accumulation as an adaptive response to mitigate oxidative stress. The COMT KO mouse has decreased placental glycogen content (Supplemental Figure 4, A and B), which at first seemed to contradict this proposed relationship between suppressed 2-ME/PlGF and increased placental glycogen. However, measurements of PlGF levels revealed that PlGF is not suppressed in COMT KO mice compared with WT (Supplemental Figure 4C). Therefore, differences in PlGF levels may partially explain the relative lack of placental glycogen in COMT KO mice compared with PlGF KO mice and human preeclampsia. Deletion of PlGF in COMT mice (DKO ${ }^{\mathrm{xDKO}}$ ) did indeed result in increased placental glycogen, lending further support to potential coordinated or overlapping roles for PlGF and 2-ME in regulating placental glycogen and metabolism in preeclampsia.

Despite significant interest in $\mathrm{PlGF}$ as a predictor and diagnostic biomarker of preeclampsia $(5,6,65)$, the precise role of $\mathrm{PlGF}$ in the placenta and preeclampsia is not well understood. A recent study by Aasa and colleagues examined the effect of PlGF loss on maternal cardiovascular physiology (23). Pregnant PlGF KO mice were found to have changes in systolic blood pressure, cardiac output, ventricular mass, and expression of cardiac 
stress-related genes in addition to a mild renal phenotype at E16. Alterations in maternal cardiac parameters occurred at E14 and E16 and normalized by E18, leading the authors to propose a limited window for PlGF activity during pregnancy with respect to maternal cardiovascular adaptations. Early transient increased uterine artery resistance was reported in PlGF KO mice; this did not have a significant impact on fetal blood flow (by umbilical artery Doppler), fetal/neonatal growth, or litter size, consistent with our litter data. Any discrepancies between our studies can likely be explained by differences in study design and methodology. We focused our evaluation late in gestation, when preeclampsia occurs most commonly; Aasa et al. highlighted a maternal phenotype that was evident at earlier time points than the ones we examined (23). Although they reported a difference in blood pressure in PlGF KO mice compared with WT C57BL/6, the absolute difference in blood pressure at the late time points of gestation was minimal and not inconsistent with our results. Variations in blood pressure may also be due to differences in genetic background of the mice. Importantly, our studies had different objectives and therefore report outcomes that are not mutually exclusive.

We acknowledge that the PlGF KO model does not faithfully recapitulate the pattern seen in preeclampsia in which PlGF is reduced but still present. The existence of $4 \mathrm{PlGF}$ splice variants in human compared with only 1 variant in mouse also suggests more complex PlGF biology and function in humans that remains to be explored in the context of preeclampsia (66). Furthermore, $\mathrm{PlGF}$ is a pleiotropic factor implicated in blood pressure regulation via the renin-angiotensin-aldosterone system $(67,68)$. This interaction was beyond the scope of our study, but may be relevant to the pathogenesis of preeclampsia $(39,69-72)$. Finally, the impact of local angiogenic factors, particularly VEGF, is an area of interest for future studies, as there is evidence that local regulation of angiogenic factors at the maternal-fetal interface is important for the maintenance of vascular integrity in the placenta (73).

Importantly, an elevated sFlt-1/PlGF ratio is not a prerequisite for the development of preeclampsia, nor is it unique to preeclampsia. As proposed in a recent review, elevated sFlt-1 and reduced PlGF levels may represent placental cellular stress or failure of homeostatic mechanisms in general, rather than preeclampsia per se (3). A similar profile of angiogenic factors is observed in a host of other conditions associated with compromised placental function (74), including fetal growth restriction $(75,76)$, fetal death $(77,78)$, multiple gestation $(79,80)$, systemic lupus erythematosus (81), and pregnancies resulting from in vitro fertilization (82). Our findings support a complex and multifaceted model for the pathogenesis of preeclampsia, consistent with the current consensus that preeclampsia is a syndrome with multiple etiologies Therefore, designating a single pathophysiologic model is not accurate (61). Therapeutic approaches have been proposed based on the idea that attenuating the antiangiogenic environment in preeclampsia will improve clinical status and delay disease progression $(13,83,84)$. However, studies revealing disease-promoting functions of PlGF (85-89) support the need for further mechanism-focused research, and therefore, interventions aiming to alter angiogenic factors in preeclampsia should be approached with caution. Here, we provide evidence for the broader impact of PlGF on placental development, potentially via regulation of fetal-placental metabolism.

\section{Methods}

Mice. PlGF KO mice (Pgf/-; mixed background, Swiss/SV129) were previously generated by deletion of exons 3-6 (16) and provided by P. Carmeliet. Homozygous adult PlGF KO and WT matings produced pregnant females carrying litters of PIGF KO and WT embryos, respectively. $\mathrm{Pgf}^{/-}$; $\mathrm{Comt}^{-/-}$DKO mice were generated by breeding COMT KO (mixed, C57BL/6/SV129) and PlGF KO (mixed, Swiss/ SV129) mice. DKO females were bred to either DKO males (DKO ${ }^{\mathrm{xDKO}}$ ). or COMT KO males $\left(\mathrm{DKO}^{\mathrm{XPIGF}}\right)$ to produce pregnancies with DKO $\left(\mathrm{Pgf}^{\prime /-} ; \mathrm{Comt}^{-/}\right)$embryos and PlGF-positive $\left(\mathrm{Pgf}^{+/-}\right.$; $\left.\mathrm{Comt}^{-/}\right)$embryos, respectively. For COMT KO experiments, pregnant COMT KO and WT mice were injected subcutaneously daily with $10 \mathrm{ng}$ 2-ME (Sigma-Aldrich) or placebo (olive oil), respectively, starting from E10, as previously described (37). The day the vaginal plug was observed was taken to be 12 hours after fertilization and set as E0.5.

ELISA, urinary protein, and blood pressure measurements. Blood and urine samples were collected from nonpregnant and pregnant PIGF KO and WT mice between E17 and E2O and from DKO and $\mathrm{DKO}^{\mathrm{xPlGF}+}$ mice at E17. Serum or plasma sFlt-1, VEGF, and PlGF levels were measured using Quantikine ELISA kits (R\&D Systems). Urine protein levels were estimated using a colorimetric assay (SigmaAldrich), as previously described (12). Blood pressure was monitored using a programmable tail-cuff sphygmomanometer (SC-1000, Hatteras Instruments), as previously described (37).

Embryo and placental measurements. Pregnant mice were sacrificed at the time points indicated above. Weights of placentas and embryos were recorded. Embryonic demise was calculated by the number of resorptions divided by the total number of embryos for each pregnancy.

Histology. Animals were perfusion fixed with $4 \%$ paraformaldehyde in $0.1 \mathrm{M}$ phosphate buffer. Paraffin-embedded organs were sectioned $(5 \mu \mathrm{m})$ and used for H\&E staining, immunohistochemistry, and immunofluorescence. H\&E-stained cross-sections from the midportion of the placenta were analyzed for trophoblast morphology and junctional zone/labyrinth architecture. Placental layers were measured to determine the relative thickness of the junctional zone and labyrinth, which was expressed as a proportion of total placental thickness. For histological analysis of kidney, randomly selected glomeruli were identified and quantified by counting the number of nuclei per 6 to 10 glomeruli per kidney. Areas of the renal corpuscle and glomerular tuft were quantified with ImageJ (NIH). The area of Bowman's space was calculated by subtracting glomerular tuft area from renal corpuscle area.

Immunohistochemistry and immunofluorescence. For immunohistochemistry, sections were deparaffinized and rehydrated, and antigen was retrieved at $98^{\circ} \mathrm{C}$ for 30 minutes in $10 \mathrm{mM}$ citrate buffer, pH 6. Sections were incubated with blocking buffer, followed by primary antibody. The following primary antibodies were used: Flt-1 (Santa Cruz Biotechnology Inc., catalog sc-9029, 1:100; Abcam, catalog ab2350, 1:100); CD34 (Abcam, catalog ab81289, 1:500). Placental Flt-1 staining was processed using a VECTASTAIN ABC Kit (Vector Laboratories). For all other stains, sections were incubated with 4plus Biotinylated Goat Anti-Rabbit Secondary Antibody and streptavidin-HRP (BioCare Medical), followed by DAB peroxidase 
substrate reagent. Sections were counterstained with hematoxylin, and $\mathrm{DAB}$ positivity was analyzed. Immunofluorescence analysis of frozen sections was performed as previously described (37). The following primary antibodies were used: Flt-1 (Abcam, catalog ab2350, 1:100); pan-cytokeratin (Sigma-Aldrich, catalog C1801, 1:200); laminin (Sigma-Aldrich, catalog L9393, 1:200); and entactin (also known as nidogen-1, Chemicon International, catalog MAB1946, 1:100). For quantification of CD34 and Flt-1, 3 visual fields were analyzed per placenta using ImageJ.

Electron microscopy of the kidney. Kidney tissues were fixed with $2.5 \%$ glutaraldehyde, $2 \%$ paraformaldehyde in $0.1 \mathrm{M}$ cacodylate buffer. Kidney segments were processed for electron microscopy and viewed with an FEI Tecnai G2 Biotwin transmission electron microscope or a JEOL 6390 scanning electron microscope, as previously described (90).

Placental glycogen measurements. Best's carmine was used to stain placental glycogen. Deparaffinized sections were incubated for 20 minutes in Best's carmine staining solution, followed by differentiation solution and dehydration. Amylase treatment was used to evaluate the specificity of Best's carmine staining of glycogen.

Glycogen content was quantified by either digestion and precipitation from placental tissue or imaging of Best's carmine-stained sections. For tissue digestion, portions of placental tissue were weighed and boiled in $30 \%$ potassium hydroxide, $5 \%$ sodium sulfate bath for 30 minutes to release the glycogen. After cooling on ice, glycogen was precipitated with 1.1 volumes of $95 \%$ ethanol. Glycogen precipitates were dissolved in water and analyzed by a phenol sulfuric acid colorimetric method (91). Calculated glycogen content was normalized to the weight of the starting tissue and expressed as $\mu \mathrm{g} / \mathrm{glycogen}$ per mg placental tissue. For quantification of glycogen cells by imaging, the number of glycogenpositive cells (by Best's carmine staining) per visual field was counted and expressed as a percentage of total cells in the junctional zone.

Human sample collection and measurement of PlGF and 2-ME levels. Blood samples from preeclampsia cases and normotensive controls were collected from patients at the University of Pennsylvania (provided by SP and JFS), Mayo Clinic (provided by VDG), and Brigham and Women's (provided by TFM) Hospitals. Preeclampsia was diagnosed according to American College of Obstetricians and Gynecologists criteria (92). PlGF levels were measured using a Quantikine ELISA Kit (R\&D Systems). Measurement of plasma 2-ME was performed by PPD Development as previously described (37). Outliers were identified using the ROUT method (robust regression and outlier removal method in GraphPad Prism 6, ref. 93); 7 outliers in the control group and 6 outliers in the PE group were identified, subsequently excluded from the data presented, and statistically analyzed, as shown in Supplemental Figure 5B. Human placenta biopsies were obtained from patients who were delivered at Brigham and Women's Hospital (patients of TFM). Placental glycogen content was quantified by the tissue digestion method described above.

Statistics. Data are presented as mean \pm SD or SEM, as indicated in the figure legends. Unpaired 2-tailed $t$ test with Welch's correction, Mann-Whitney $U$ test, or 1-way ANOVA with Tukey's multiple comparisons test was used as appropriate and specified in the figure legends to determine significance. Statistical significance was defined as $P<0.05$. GraphPad Prism 6 software was used for statistical analyses.

Study approval. All animal experiments were approved by the Beth Israel Deaconess Medical Center Institutional Animal Care and Use Committee. Studies involving human specimens were approved by the University of Pennsylvania, Mayo Clinic, and Brigham and Women's Hospital Institutional Review Boards. Written, informed consent was obtained from subjects prior to inclusion in the study.

\section{Author contributions}

JGP analyzed data, generated figures, and wrote the manuscript. $\mathrm{KK}$ and MK designed and performed experiments with DKO and COMT KO mice and contributed to Figures 1, 3, and 4 and Supplemental Figures 4-6. HS contributed to Figures 1-4 and Supplemental Figures 1-3. LX performed experiments with PlGF KO mice and contributed to Figures 1-3. YH contributed to Supplemental Figure 5. SBL contributed to Figure 4 and Supplemental Figure 5. VHG performed electron microscopy in Figure 2. SP, JFS, VDG, and TFM provided human specimens. PC provided PlGF KO mice. JFS, KHL, BMS, and PC provided intellectual input. VSL provided intellectual input and edited figures and manuscript. RK conceptually designed the strategy for this study, provided intellectual input, and helped write the manuscript.

\section{Acknowledgments}

This work was supported in part by funds provided by the MD Anderson Cancer Center, the Cancer Prevention and Research Institute of Texas, and the University of Texas STARs Program. This study was also supported by funding from a Preeclampsia Foundation Vision Grant and the Baylor College of Medicine Maternal-Fetal Medicine Fellowship (to JGP).

Address correspondence to: Raghu Kalluri, Department of Cancer Biology, Metastasis Research Center, University of Texas MD Anderson Cancer Center, 1881 East Road, Unit 1906, Houston, Texas 77054, USA. Phone: 713.794.5310; Email: rkalluri@mdanderson.org.

KK's present address is: Department of Diabetology and Endocrinology, Kanazawa Medical University, Kanazawa, Japan.

MK's present address is: Takeda Hospital, Kyoto, Japan.

LX's present address is: China R\&D and Scientific Affairs, Jassen (China) Research \& Development Center, Shanghai, China.

YH's present address is: Departments of Nephrology and Internal Medicine, Sanai Memorial Soga Hospital, Chiba, Japan.

SBL's present address is: Department of Internal Medicine, Pusan National University School of Medicine, Yangsan, South Korea.
1. Chaiworapongsa T, Chaemsaithong P, Yeo L, Romero R. Pre-eclampsia part 1: current understanding of its pathophysiology. Nat Rev Nephrol. 2014;10(8):466-480.
2. Fisher SJ. Why is placentation abnormal in preeclampsia? Am J Obstet Gynecol. 2015;213(4 Suppl):S115-S122.

3. Redman CW, Staff AC. Preeclampsia, biomark- ers, syncytiotrophoblast stress, and placental capacity. Am J Obstet Gynecol. 2015;213(4 Suppl):S9.e1-S9.e4.

4. Brosens I, Pijnenborg R, Vercruysse L, Romero R. 
The "great obstetrical syndromes" are associated with disorders of deep placentation. Am JObstet Gynecol. 2011;204(3):193-201.

5. Zeisler $\mathrm{H}$, et al. Predictive value of the sFlt-1:PlGF ratio in women with suspected preeclampsia. N Engl J Med. 2016;374(1):13-22.

6. Chappell LC, et al. Diagnostic accuracy of placental growth factor in women with suspected preeclampsia: a prospective multicenter study. Circulation. 2013;128(19):2121-2131.

7. Andrietti S, Silva M, Wright A, Wright D, Nicolaides $\mathrm{KH}$. Competing-risks model in screening for pre-eclampsia by maternal factors and biomarkers at 35-37 weeks' gestation. Ultrasound Obstet Gynecol. 2016;48(1):72-79.

8. Schiettecatte J, et al. Multicenter evaluation of the first automated Elecsys sFlt- 1 and PlGF assays in normal pregnancies and preeclampsia. Clin Biochem. 2010;43(9):768-770.

9. Hiratsuka S, Minowa O, Kuno J, Noda T, Shibuya M. Flt-1 lacking the tyrosine kinase domain is sufficient for normal development and angiogenesis in mice. Proc Natl Acad Sci US A. 1998;95(16):9349-9354.

10. Levine RJ, et al. Circulating angiogenic factors and the risk of preeclampsia. N Engl J Med. 2004;350(7):672-683

11. Maynard SE, et al. Excess placental soluble fms-like tyrosine kinase 1 (sFlt1) may contribute to endothelial dysfunction, hypertension, and proteinuria in preeclampsia. JClin Invest. 2003;111(5):649-658

12. Sugimoto $\mathrm{H}$, et al. Neutralization of circulating vascular endothelial growth factor (VEGF) by anti-VEGF antibodies and soluble VEGF receptor 1 (sFlt-1) induces proteinuria. J Biol Chem. 2003;278(15):12605-12608.

13. Kumasawa K, et al. Pravastatin induces placental growth factor (PGF) and ameliorates preeclampsia in a mouse model. Proc Natl Acad Sci U S A. 2011;108(4):1451-1455.

14. Cerdeira AS, Karumanchi SA. Angiogenic factors in preeclampsia and related disorders. Cold Spring Harb Perspect Med. 2012;2(11):a006585.

15. Maglione D, Guerriero V, Viglietto G, Delli-Bovi $\mathrm{P}$, Persico MG. Isolation of a human placenta cDNA coding for a protein related to the vascular permeability factor. Proc Natl Acad Sci U S A. 1991;88(20):9267-9271.

16. Carmeliet $P$, et al. Synergism between vascular endothelial growth factor and placental growth factor contributes to angiogenesis and plasma extravasation in pathological conditions. Nat Med. 2001;7(5):575-583.

17. Park JE, Chen HH, Winer J, Houck KA, Ferrara N. Placenta growth factor. Potentiation of vascular endothelial growth factor bioactivity, in vitro and in vivo, and high affinity binding to Flt-1 but not to Flk-1/KDR. J Biol Chem . 1994;269(41):25646-25654

18. Ferrara N, et al. Heterozygous embryonic lethality induced by targeted inactivation of the VEGF gene. Nature. 1996;380(6573):439-442.

19. Dewerchin M, Carmeliet P. PlGF: a multitasking cytokine with disease-restricted activity. Cold Spring Harb Perspect Med. 2012;2(8):a011056.

20. Fischer C, Mazzone M, Jonckx B, Carmeliet P. FLT1 and its ligands VEGFB and PlGF: drug tar- gets for anti-angiogenic therapy? Nat Rev Cancer 2008;8(12):942-956.

21. Tayade C, et al. Genetic deletion of placenta growth factor in mice alters uterine NK cells. Jimmunol. 2007;178(7):4267-4275.

22. Saffer $\mathrm{C}$, et al. Determination of placental growth factor (PlGF) levels in healthy pregnant women without signs or symptoms of preeclampsia. Pregnancy Hypertens. 2013;3(2):124-132.

23. Aasa KL, et al. Placental growth factor influences maternal cardiovascular adaptation to pregnancy in mice. Biol Reprod. 2015;92(2):44.

24. Achen MG, Gad JM, Stacker SA, Wilks AF. Placenta growth factor and vascular endothelial growth factor are co-expressed during early embryonic development. Growth Factors. 1997;15(1):69-80.

25. Luna RL, et al. Placental growth factor deficiency is associated with impaired cerebra vascular development in mice. Mol Hum Reprod. 2016;22(2):130-142.

26. Jobe SO, Tyler CT, Magness RR. Aberrant synthesis, metabolism, and plasma accumulation of circulating estrogens and estrogen metabolites in preeclampsia implications for vascular dysfunction. Hypertension. 2013;61(2):480-487.

27. Wantania J, Attamimi A, Siswishanto R. A Compar ison of 2-Methoxyestradiol Value in Women with Severe Preeclampsia Versus Normotensive Pregnancy. JClin Diagn Res. 2017;11(3):QC35-QC38.

28. Zhang Y, Wang T, Shen Y, Wang X, Baker PN, Zhao A. 2-Methoxyestradiol deficiency is strongly related to hypertension in early onset severe pre-eclampsia. Pregnancy Hypertens. 2014;4(3):215-219.

29. Shen Z, et al. Decreased maternal serum 2-methoxyestradiol levels are associated with the development of preeclampsia. Cell Physiol Biochem. 2014;34(6):2189-2199.

30. Barnea ER, MacLusky NJ, DeCherney AH, Naftolin F. Catechol-o-methyl transferase activity in the human term placenta. Am J Perinatol. 1988;5(2):121-127.

31. Pertegal M, et al. 2-methoxyestradiol plasma levels are associated with clinical severity indices and biomarkers of preeclampsia. Reprod Sci. 2015;22(2):198-206.

32. Pérez-Sepúlveda A, et al. Low 2-methoxyestradio levels at the first trimester of pregnancy are associated with the development of pre-eclampsia. Prenat Diagn. 2012;32(11):1053-1058.

33. Lee SB, et al. Preeclampsia: 2-methoxyestradio induces cytotrophoblast invasion and vascular development specifically under hypoxic conditions. Am J Pathol. 2010;176(2):710-720.

34. Mabjeesh NJ, et al. 2ME2 inhibits tumor growth and angiogenesis by disrupting microtubules and dysregulating HIF. Cancer Cell. 2003;3(4):363-375.

35. Becker CM, et al. 2-methoxyestradiol inhibits hypoxia-inducible factor-1 $\{$ alpha\} and suppresses growth of lesions in a mouse model of endometriosis. Am J Pathol. 2008;172(2):534-544.

36. Lee DK, Nevo O. 2-Methoxyestradiol regulates VEGFR-2 and sFlt-1 expression in human placenta. Placenta. 2015;36(2):125-130.

37. Kanasaki K, et al. Deficiency in catechol-Omethyltransferase and 2-methoxyoestradiol is associated with pre-eclampsia. Nature.
2008;453(7198):1117-1121.

38. Stanley JL, et al. Sildenafil citrate rescues fetal growth in the catechol-O-methyl transferase knockout mouse model. Hypertension. 2012;59(5):1021-1028.

39. Ueki N, Kanasaki K, Kanasaki M, Takeda S, Koya D. Catechol-O-methyltransferase deficiency leads to hypersensitivity of the pressor response against angiotensin II. Hypertension. 2017;69(6):1156-1164.

40. Stanley JL, et al. Sildenafil therapy normalizes the aberrant metabolomic profile in the $\mathrm{Comt}^{(-/)}$ mouse model of preeclampsia/fetal growth restriction. Sci Rep. 2015;5:18241.

41. Arkwright PD, Rademacher TW, Dwek RA, Redman CW. Pre-eclampsia is associated with an increase in trophoblast glycogen content and glycogen synthase activity, similar to that found in hydatidiform moles. J Clin Invest. 1993;91(6):2744-2753

42. Robb SA, Hytten FE. Placental glycogen. Br J Obstet Gynaecol. 1976;83(1):43-53.

43. Bloxam DL, Bullen BE, Walters BN, Lao TT. Placental glycolysis and energy metabolism in preeclampsia. Am JObstet Gynecol. 1987;157(1):97-101.

44. Fox H. Basement membrane changes in the villi of the human placenta. JObstet Gynaecol Br Commonw. 1968;75(3):302-306.

45. Castro EC, Reis MA, Teixeira VP. Thickening of the amnion basement membrane and its relationship to placental inflammatory lesions and fetal and maternal disorders. Eur J Obstet Gynecol Reprod Biol. 2004;114(2):171-176

46. Robinson CJ, Johnson DD, Chang EY, Armstrong DM, Wang W. Evaluation of placenta growth factor and soluble Fms-like tyrosine kinase 1 receptor levels in mild and severe preeclampsia. Am JObstet Gynecol. 2006;195(1):255-259.

47. Verlohren S, et al. The sFlt-1/PlGF ratio in different types of hypertensive pregnancy disorders and its prognostic potential in preeclamptic patients. Am JObstet Gynecol. 2012;206(1):58.e1-58.e8.

48. Moore Simas TA, Crawford SL, Solitro MJ, Frost SC, Meyer BA, Maynard SE. Angiogenic factors for the prediction of preeclampsia in high-risk women. Am JObstet Gynecol. 2007;197(3):244.e1-244.e8.

49. Kusanovic JP, et al. A prospective cohort study of the value of maternal plasma concentrations of angiogenic and anti-angiogenic factors in early pregnancy and midtrimester in the identification of patients destined to develop preeclampsia.J Matern Fetal Neonatal Med. 2009;22(11):1021-1038.

50. Rai A, Cross JC. Development of the hemochorial maternal vascular spaces in the placenta through endothelial and vasculogenic mimicry. Dev Biol. 2014;387(2):131-141.

51. Akison LK, Nitert MD, Clifton VL, Moritz KM, Simmons DG. Review: Alterations in placental glycogen deposition in complicated pregnancies: Current preclinical and clinical evidence. Placenta. 2017;54:52-58.

52. Coan PM, Conroy N, Burton GJ, Ferguson-Smith AC. Origin and characteristics of glycogen cells in the developing murine placenta. Dev Dyn. 2006;235(12):3280-3294.

53. Tunster SJ, McNamara GI, Creeth HDJ, John RM. Increased dosage of the imprinted Ascl2 gene restrains two key endocrine lineages of the 
mouse Placenta. Dev Biol. 2016;418(1):55-65.

54. Tunster SJ, Tycko B, John RM. The imprinted Phlda2 gene regulates extraembryonic energy stores. Mol Cell Biol. 2010;30(1):295-306.

55. Longo LD, Yuen P, Gusseck DJ. Anaerobic, glycogen-dependent transport of amino acids by the placenta. Nature. 1973;243(5409):531-533.

56. Gebbe SG, Demers LM, Greep RO, Villee CA. The effects of hypoxia on placental glycogen metabolism. Am J Obstet Gynecol. 1972;114(4):540-545.

57. Cheng KW, et al. Rab25 increases cellular ATP and glycogen stores protecting cancer cells from bioenergetic stress. EMBO Mol Med. 2012;4(2):125-141.

58. Hayward CE, et al. Placental adaptation: what can we learn from birthweight:placental weight ratio? Front Physiol. 2016;7:28.

59. Aye IL, Rosario FJ, Powell TL, Jansson T. Reply to Carbillon: Fetal/placental weight ratio and placental function. Proc Natl Acad Sci U S A. 2016;113(3):E261.

60. Hirashima M, Lu Y, Byers L, Rossant J. Trophoblast expression of fms-like tyrosine kinase 1 is not required for the establishment of the maternalfetal interface in the mouse placenta. Proc Natl Acad Sci U S A. 2003;100(26):15637-15642.

61. American College of Obstetricians Gynecologists, Task Force on Hypertension in Pregnancy. Hypertension in pregnancy. Report of the American College of Obstetricians and Gynecologists' Task Force on Hypertension in Pregnancy. Obstet Gynecol. 2013;122(5):1122-1131.

62. Shenoy V, Kanasaki K, Kalluri R. Pre-eclampsia: connecting angiogenic and metabolic pathways. Trends Endocrinol Metab. 2010;21(9):529-536.

63. Hill LD, et al. Epistasis between COMT and MTHFR in maternal-fetal dyads increases risk for preeclampsia. PLoS One. 2011;6(1):e16681.

64. Perez-Sepulveda A, España-Perrot PP, Norwitz ER, Illanes SE. Metabolic pathways involved in 2-methoxyestradiol synthesis and their role in preeclampsia. Reprod Sci. 2013;20(9):1020-1029.

65. Schiettecatte J, et al. Multicenter evaluation of the first automated Elecsys sFlt-1 and PlGF assays in normal pregnancies and preeclampsia. Clin Biochem. 2010;43(9):768-770.

66. Bates DO. An unexpected tail of VEGF and PlGF in pre-eclampsia. Biochem Soc Trans. 2011;39(6):1576-1582.

67. Carnevale D, et al. The angiogenic factor PlGF mediates a neuroimmune interaction in the spleen to allow the onset of hypertension. Immunity. 2014;41(5):737-752.

68. Jaffe IZ, et al. Placental growth factor mediates aldosterone-dependent vascular injury in mice. J Clin Invest. 2010;120(11):3891-3900.

69. Gant NF, Whalley PJ, Everett RB, Worley RJ, MacDonald PC. Control of vascular reactivity in pregnancy. Am J Kidney Dis. 1987;9(4):303-307.

70. Wallukat G, et al. Patients with preeclampsia develop agonistic autoantibodies against the angiotensin AT1 receptor. J Clin Invest. 1999;103(7):945-952.

71. Siddiqui AH, Irani RA, Blackwell SC, Ramin SM, Kellems RE, Xia Y. Angiotensin receptor agonistic autoantibody is highly prevalent in preeclampsia: correlation with disease severity. Hypertension. 2010;55(2):386-393.

72. Gormley M, Ona K, Kapidzic M, GarridoGomez T, Zdravkovic T, Fisher SJ. Preeclampsia: novel insights from global RNA profiling of trophoblast subpopulations. Am JObstet Gynecol. 2017;217(2):200.e1-200.e17.

73. Fan X, et al. Endometrial VEGF induces placental sFLT1 and leads to pregnancy complications. J Clin Invest. 2014;124(11):4941-4952.

74. Korzeniewski SJ, et al. Maternal plasma angiogenic index-1 (placental growth factor/soluble vascular endothelial growth factor receptor-1) is a biomarker for the burden of placental lesions consistent with uteroplacental underperfusion: a longitudinal case-cohort study. Am JObstet Gynecol. 2016;214(5):629.e1-629.e17.

75. Savvidou MD, Yu CK, Harland LC, Hingorani AD, Nicolaides KH. Maternal serum concentration of soluble fms-like tyrosine kinase 1 and vascular endothelial growth factor in women with abnormal uterine artery Doppler and in those with fetal growth restriction. Am JObstet Gynecol. 2006;195(6):1668-1673.

76. Chaiworapongsa T, et al. The maternal plasma soluble vascular endothelial growth factor receptor-1 concentration is elevated in SGA and the magnitude of the increase relates to Doppler abnormalities in the maternal and fetal circulation. J Matern Fetal Neonatal Med. 2008;21(1):25-40.

77. Espinoza J, et al. Unexplained fetal death: another anti-angiogenic state. JMatern Fetal Neonatal Med. 2007;20(7):495-507.

78. Romero R, et al. An imbalance between angiogenic and anti-angiogenic factors precedes fetal death in a subset of patients: results of a longitudinal study. JMatern Fetal Neonatal Med. 2010;23(12):1384-1399.

79. Dröge L, et al. Maternal serum sFlt-1/PlGF ratio in twin pregnancies with and without pre-eclampsia in comparison with singleton pregnancies. Ultrasound Obstet Gynecol. 2015;45(3):286-293.
80. Faupel-Badger JM, et al. Maternal circulating angiogenic factors in twin and singleton pregnancies. Am JObstet Gynecol. 2015;212(5):636.e1-636.e8.

81. Kim MY, et al. Angiogenic factor imbalance early in pregnancy predicts adverse outcomes in patients with lupus and antiphospholipid antibodies: results of the PROMISSE study. Am J Obstet Gynecol. 2016;214(1):108.e1-108.e14.

82. Lee MS, et al. Angiogenic markers in pregnancies conceived through in vitro fertilization. $A m \mathrm{~J}$ Obstet Gynecol. 2015;213(2):212.e1-212.e8.

83. Spradley FT, et al. Placental growth factor administration abolishes placental ischemia-induced hypertension. Hypertension. 2016;67(4):740-747.

84. Thadhani R, et al. Removal of soluble Fms-like tyrosine kinase-1 by dextran sulfate apheresis in preeclampsia. JAm Soc Nephrol. 2016;27(3):903-913.

85. Patel N, Tahara SM, Malik P, Kalra VK. Involvement of miR-30c and miR-301a in immediate induction of plasminogen activator inhibitor-1 by placental growth factor in human pulmonary endothelial cells. Biochem J. 2011;434(3):473-482.

86. Khurana $\mathrm{R}$, et al. Placental growth factor promotes atherosclerotic intimal thickening and macrophage accumulation. Circulation. 2005;111(21):2828-2836.

87. Roncal C, et al. Short-term delivery of anti-PlGF antibody delays progression of atherosclerotic plaques to vulnerable lesions. Cardiovasc Res. 2010;86(1):29-36.

88. Sundaram N, et al. High levels of placenta growth factor in sickle cell disease promote pulmonary hypertension. Blood. 2010;116(1):109-112.

89. Tsao PN, et al. Overexpression of placenta growth factor contributes to the pathogenesis of pulmonary emphysema. Am J Respir Crit Care Med. 2004;169(4):505-511.

90. Cosgrove D, et al. Integrin alpha1beta1 and transforming growth factor-beta1 play distinct roles in alport glomerular pathogenesis and serve as dual targets for metabolic therapy. Am J Pathol. 2000;157(5):1649-1659.

91. Lo S, Russell JC, Taylor AW. Determination of glycogen in small tissue samples. J Appl Physiol. 1970;28(2):234-236.

92. ACOG Committee on Practice Bulletins-Obstetrics. ACOG practice bulletin. Diagnosis and management of preeclampsia and eclampsia. Number 33, January 2002. Obstet Gynecol. 2002;99(1):159-167.

93. Motulsky HJ, Brown RE. Detecting ouliers when fitting data with nonlinear regression - a new method based on robust nonlinear regression and the false discover rate. BMC Bioinformatics. 2006;7:123. 